Review Article

Open Access

\title{
Type 1 Diabetes Glucose Control related Home-Based Testing: A Mini-Review of Tools and Patient Knowledge and Implementation
}

\author{
Alicia J Jenkins ${ }^{1-3}$, Gary Kilov ${ }^{4,5}$, Emma Scott ${ }^{1,6}$, Greg R Fulcher ${ }^{6}$, David N O'Neal ${ }^{1-3}$ and Andrzej S Januszewski ${ }^{1,3 *}$ \\ ${ }^{I}$ NHMRC Clinical Trials Centre, The University of Sydney, Australia \\ ${ }^{2}$ Department of Diabetes and Endocrinology, St. Vincent's Hospital Melbourne, Australia \\ ${ }^{3}$ Department of Medicine, University of Melbourne, Australia \\ ${ }^{4}$ Seaport Diabetes incorporated within Seaport Practice, Australia \\ ${ }^{5}$ Department of General Practice, University of Melbourne, Australia \\ ${ }^{6}$ Department of Endocrinology, Royal North Shore Hospital, The University of Sydney, Australia
}

\section{Article Info}

\section{*Corresponding author:}

Andrzej S Januszewski

NHMRC Clinical Trials Centre

Level 6, Medical Foundation Building

92 - 94 Parramatta Road

Camper down, NSW, 2050

Australia

Tel: +61295625000

Fax: +61295625090

E-mail: andrzej.januszewski@ctc.usyd.edu.au

\section{Received: June 5, 2018}

Accepted: July 16, 2018

Published: July 20, 2018

Citation: Jenkins AJ, Kilov G, Scott E, Fulcher GR, O'Neal DN, Januszewski AS. Type 1 Diabetes Glucose Control related HomeBased Testing: A Mini-Review of Tools and Patient Knowledge and Implementation. Madridge J Diabetes. 2018; 2(2): 55-62. doi: $10.18689 / \mathrm{mjd}-100011$

Copyright: $\odot 2018$ The Author(s). This work is licensed under a Creative Commons Attribution 4.0 International License, which permits unrestricted use, distribution, and reproduction in any medium, provided the original work is properly cited.

Published by Madridge Publishers

\begin{abstract}
Background: In Type 1 diabetes (T1D) glycaemic control is key to the prevention of acute and chronic complications. An increasing array of devices is available for at-home monitoring. Tools must be used appropriately, and the information gained utilised well to maximise clinical benefit.

Aims: This mini-review describes clinically available home glucose and ketone monitoring tools and discusses factors which impact glucose outcomes. Additionally, findings from the authors' surveys of T1D adults regarding glucose self-care are discussed.

Results: Blood glucose strips and meters and urine glucose strips have been available for decades. More recently available are continuous glucose monitors (CGM) and flash glucose monitoring (FGM) which assess interstitial fluid glucose levels. Blood and urine ketone test-strips are also available. In surveys of adults with T1D in two tertiary referral diabetes clinics $(n=205)$ and in general practice $(G P, n=35)$ self-reported rates of blood glucose (BG) monitoring and glucose targets were similar, with a mean (SD) of 5.3 (2.5) tests/day. Deficits in knowledge or practice related to the care of hypothetical pre-bed BG levels and of metabolic control around illness, exercise or alcohol were common. Only about one third reported treating nocturnal hypoglycaemia with the recommended refined then complex carbohydrate. Only $66 \%$ reported having in-date ketone teststrips at home. Further education was often desired, but difficult to predict based on demographics.
\end{abstract}

Conclusions: A range of tools are available to test glucose and ketone levels at home. Many adults with T1D have suboptimal knowledge and behaviour regarding glucose self-management.

Keywords: Type 1 Diabetes; Glycaemic control; Self-Management; Diabetes Education.

\section{Introduction}

The incidence and prevalence of diabetes, including Type 1 diabetes (T1D) are increasing globally [1], even in low-incidence countries [2]. Glucose control is a daily challenge, and its optimisation is key to the prevention of the acute and chronic complications, which are costly both personally and to the healthcare system. There are 
limited data regarding patient knowledge and behaviour related to glucose control, including high risk nocturnal hypoglycaemia prevention and care. Most adults with T1D spend only $\approx 2-3$-hours a year with their diabetes care-team, therefore glucose self-management is essential. Patient time with clinicians is often limited; hence tools which can facilitate self-care assessment are desirable.

The purpose of this mini-review is to describe currently clinically available types of glucose and ketone monitoring, and to review the findings from our novel recently (published) surveys of T1D adults and their glucose-related self-care practices. As this is a rapidly evolving area, and different brands and models of devices are available in different regions, this article focuses on types of technologies and gives examples of clinically available brands, particularly citing the first, or sometimes only, clinically available system of its type.

\section{Type 1 Diabetes and the Importance of Glucose Control}

As shown by the Diabetes Control and Complications Trial (DCCT) and its observational follow-up, the Epidemiology of Diabetes Interventions and Complications (EDIC) Study, higher $\mathrm{HbA1c}$ levels are associated with increased risk of diabetic retinopathy, nephropathy and neuropathy and intensive diabetes management leading to $\approx 2 \%$ lower $\mathrm{HbA} 1 \mathrm{c}$ levels significantly reduces all microvascular complications and subsequent cardiovascular disease events [3]. The DCCT/ EDIC study also demonstrated 'metabolic memory' for glycaemia, that is the persistence of the body's tissues responses to good or poor glucose control for many years after the resolution of that good or poor glucose control [4, 5]. The recommended $\mathrm{HbA} 1 \mathrm{c}$ level for most adults with $\mathrm{T1D}$ is $<7 \%[6,7]$. Unfortunately, the mean (SD) HbA1c in Australians with T1D is $8.5(1.8) \%$, with less than $20 \%$ achieving the recommended target [8].

In affluent countries self-monitoring of capillary blood glucose (BG) levels at home is common with recommendations for adults with T1D to test at least four times a day, ideally premeals and pre-bed, to confirm hypoglycaemia and recovery from hypoglycaemia, and prior to insulin dosing for meals or high glucose correction [9]. More frequent BG testing is recommended during sick days [9] or other circumstances such as during and after endurance exercise, travels crossing time-zones and in those with impaired hypoglycaemia awareness. More recently available interstitial fluid glucose monitoring, if affordable and acceptable to the user, provides far more frequent assessments of glucose levels (up to 288 times a day with systems providing glucose levels every 5 minutes), albeit often with differences in absolute values and a time lag relative to glucose levels in blood (discussed below).

The acute complications of T1D include hypoglycaemia, hyperglycaemia and diabetic ketoacidosis (DKA). Poor glucose control is also often associated with increased risk of infections and with mental well-being issues.

\section{Hypoglycaemia}

The average person with T1D in Australia has two to three mild episodes of hypoglycaemia per week, and one episode per year of severe hypoglycaemia (defined as that requiring assistance from someone else for recovery) [10]. About 30\% of people with T1D will experience impaired hypoglycaemia awareness at some stage, which is associated with a 6-fold increased risk of severe hypoglycaemia [10, 11]. Nocturnal hypoglycaemia is particularly likely to be unrecognised by the T1D patient, as sleep (and also exercise and recent hypoglycaemia) impair counter-regulatory responses [12]. Hypoglycaemia can cause seizures, cardiac arrhythmias and death, including the so-called 'dead in bed' syndrome in which an otherwise well person with T1D goes to bed and is found dead next morning, with no explanatory findings on autopsy $[13,14]$. Hypoglycaemia-induced cardiac QT prolongation and cardiac arrhythmia is thought to be the cause. Simultaneous CGM and Holter monitor studies have documented frequent associations between low glucose levels and ECG abnormalities, including atrial and ventricular rhythm disturbances (15).

\section{Glucose Variability}

More recently, interest in glucose variability (GV) has increased. GV can be measured in the short- term (based on glucose fluctuations across days) usually by continuous glucose monitoring (CGM) or flash glucose monitoring (FGM) or by repeated fingerprick blood glucose $(B G)$ levels, or in the longterm, over months to years, based on HbA1c levels. Greater GV has been associated with reduced quality of life, increased risk of mild and severe hypoglycaemia, vascular dysfunction, increased oxidative stress and inflammation and vascular complications [16-19]. As yet there is no consensus with regard to recommended targets for all the various measures of GV.

\section{Tools for Blood-based Glucose Control Assessment}

The commonest laboratory tool to assess glucose control is $\mathrm{HbA} 1 \mathrm{c}$, which reflects average glucose levels over the previous 2-3 months and is usually the major glucose treatment target recommended in national guidelines $[6,7,18]$. HbA1c results can be interfered with by haemoglobinopathies, anaemia and ethnicity [20].

The frequency of home BG monitoring has been inversely correlated with HbA1c levels $[9,21]$, but of course the test itself is not the treatment, but rather a tool to guide adjustments to insulin doses, diet or physical activity, or use of adjunct glucose lowering drugs.

\section{Blood or Urine Ketone Tests at Home}

Blood or urine ketone measurements are also recommended during sick days and if BG levels are $15 \mathrm{mmmol} / \mathrm{l}$ or more, particularly if elevated for several hours [22]. Blood and urine glucose and ketone test strips are available, though blood teststrips are preferred for several reasons: patient preference, 
because blood tests are more quantitative than urine tests, because urine levels lag behind blood levels time-wise, and the renal threshold for glucose loss may vary considerably between and within individuals [23].

\section{Interstitial Fluid Glucose Testing Systems}

More recently available are interstitial fluid glucose testing systems which can measure and report interstitial fluid glucose levels (depending on the CGM or FGM system used) every $1-15$ mins for $6,7,10$ or 14 days. There are both realtime (RT) and masked CGM systems.

\section{Real-time (RT)-CGM}

With the real-time systems the glucose data are immediately available to the wearer. The RT-CGM systems can provide auditory or vibratory alerts for (actual or sometimes predicted) high or low glucose levels and rapid glucose changes, and some systems (for example, the Dexcom G5 or G6 ${ }^{\mathrm{TM}}$ and Guardian Connect $\left.{ }^{\mathrm{TM}}\right)$, results and alerts can also be shared real-time with a third party, such as a parent or carer [24-28]. These CGM systems usually require calibration by BG testing twice a day (in each 12-hour block). Relative to BG monitoring, meta-analyses of RT-CGM trials demonstrate $\mathrm{HbA} 1 \mathrm{c}$ reductions by about $0.25 \%$ with their use, with similar $\mathrm{HbA} 1 \mathrm{c}$ benefit if used with multiple daily insulin injections (MDI) or an insulin pump [29].

\section{Masked CGM}

The masked CGM systems, in which glucose results are not available to the wearer and clinical assessment are of value as a research educational tool. The sensor device is usually inserted, removed and data down-loaded by the clinician, and are commonly used to assess glucose control in people with Type 2 diabetes [26, 28, 30] and gestational diabetes [29, 31]. Both masked and RT-CGM systems can be downloaded for review and sharing. As the masked CGM data are only available retrospectively, the results cannot be responded to by the user in real-time and there are no glucose related alerts or sharing real-time with a third party.

\section{RT-CGM Systems that can Modulate Insulin Delivery}

Some commercial RT-CGM systems (currently in Australia only Medtronic) can be linked with insulin pumps and modulate insulin delivery. Depending on which insulin pump model is used with RT-CGM, the system can cease insulin delivery at or before a specified low interstitial fluid glucose level and restart insulin delivery on patient initiation, automatically after 2-hours, or when the interstitial glucose level has reached a specified higher level [32-34]. Different glucose alerts, insulin suspend and restart levels can be set for different times of day.

Trials of RT-CGM systems with low glucose suspend (LGS) or predictive LGS options have been shown to improve $\mathrm{HbA} 1 \mathrm{c}$ levels (by about $0.25 \%$ ) for insulin pump users, and also increase time in target glucose ranges and reduce hypoglycaemia, in particular severe hypoglycaemia, and low glucose time [31-33]. Continuous use of RT-CGM in pregnant women with T1D using either an insulin pump or MDI has been shown to significantly improve $\mathrm{HbA} 1 \mathrm{c}$ levels, time in glucose target range, GV, and neonatal health outcomes, with major reductions in large for gestation age babies, intensive care admissions, neonatal hypoglycaemia and length of hospital stay [35].

In 2017 Medtronic released its (Medtronic 670G) hybrid closed loop (HCL) insulin pump and RT- CGM system in the USA, which is currently the only commercial clinically available $\mathrm{HCL}$ system. Release in other countries is anticipated soon. The system is referred to as a hybrid closed loop pump as user interactions are still required. In this system RT-CGM results and an in-built personalised control algorithm guide insulin delivery with microboluses every few minutes rather than flat basal rates for several hours [36], although the bolus nature of insulin delivery would be dampened by its subcutaneous delivery and absorption. Such systems still require home BG testing, such as to calibrate the CGM, and for meal and correction boluses. In its 3-month pivotal trial $(n=129$, aged 14 - 75 years) use of the Medtronic $\mathrm{HCL}$ system reduced $\mathrm{HbA1c}$ levels by $\approx 0.5 \%$, significantly increased time in glucose target range $(3.9-10 \mathrm{mmol} / \mathrm{l})$ to $\approx 70 \%$ of the day, and significantly reduced low glucose time. There were no episodes of DKA or severe hypoglycaemia [35]. Meta-analyses of trials of artificial pancreas systems versus clinically available (first and second generation) pumps and RT-CGM systems demonstrate time in glucose target range of $70-85 \%$, with $12.6 \%$ more time in target range and $50 \%$ less time $<3.9 \mathrm{mmol} / \mathrm{l}$ (37). Limitations of the $\mathrm{HCL}$ system are that the CGM data and the automatic insulin delivery functions are not always available, such as when the glucose sensor signal is lost, glucose levels are outside range or (as a safety feature) when there are high insulin delivery rates. Other limitations are that the system still requires user input such as for meal boluses, exercise and at unexpected times [36] and some would prefer a more aggressive glucose control algorithm. These systems will likely improve with new versions.

\section{Flash glucose monitoring (FGM)}

FGM measures interstitial fluid glucose levels every 15-minutes, but only delivers results when the glucose sensor / transmitter is 'flashed' or 'swiped' by the specialised (Abbott) meter, which also can be used with blood glucose and ketone test-strips. The sensor, which can transmit a signal through clothing more recently a compatible smartphone can be used (up to $4 \mathrm{~cm}$ ), can last up to 14-days and works best when placed on the upper arm. As well as the current interstitial fluid glucose level the system provides trend arrows for rising or falling glucose levels and a graph of the last 8-hours of interstitial fluid glucose readings. As the system is factory calibrated no BG calibrations by the user are required. There are no currently no glucose alerts, real-time glucose data sharing, nor can it be linked with insulin pumps to modulate insulin delivery [38-40]. FGM approvals differ between countries, and include approvals for adults and children, T1D and insulin treated Type 2 diabetes (T2D), and (although not in Australia) for insulin dosing without BG checking. In a large multi-country real-world (not clinical trial) study of T1D and 
T2D ( $n=50,831$ readers and 279,446 glucose sensors) the mean number of scans per day was 16.3 per user [41]. A higher frequency of glucose checking using FGM was associated with lower estimated $\mathrm{HbA} 1 \mathrm{c}$ levels, more time in target glucose range and less low and high glucose time [42].

\section{Limitations of CGM and FGM}

Limitations of CGM and FGM Include the need for patients to wear a device (continuously) and for CGM, but not FGM, for twice daily BG calibrations. The amount of glucose information can be excessive for some, increasing distress related to the time out of target range and glucose variability. If too frequent or too large insulin boluses are given to reduce high glucose levels there is potential for insulin stacking and hypoglycaemia. There are often differences between blood and interstitial fluid glucose values related to a lag (behind blood) of about 10 minutes and differences in absolute values of about $10 \%$, usually with larger differences at glucose extremes, with even greater divergence between interstitial fluid and blood glucose levels if the FGM is somewhere else on the body than the recommended upper arm site [41]. Some oral substances can affect CGM or FGM readings. Oral paracetamol can increase CGM sensor (but not BG test-strip levels) by up to $25 \%$ for up to 8-hours [43]. Whilst the FGM sensor claims not to be affected by paracetamol, salicyclic acid and ascorbic acid may interfere, and as yet FGM has not been evaluated for dialysis patients [44]. Both CGM and FGM have operating temperature and altitude sensor ranges, but most users are within the usual limits. For people using such systems at extremes the specifications of their specific device should be checked. Similarly, while most currently available sensors are suitable for bathing, showering or swimming, the suitability of each specific device for prolonged exposure to water, deep water diver or sea water immersion should be checked.

There are additional time and educational requirements for both patients and clinicians related to CGM or FGM use. In most countries, CGM and FGM are not fully subsidised by the Government or Private Health Insurance companies hence the out-of-pocket costs for consumers to purchase a transmitter and scanner can be substantial. In Australia the standard cost per (disposable 6-14 day sensor) ranges $\$ 75$ $\$ 92$. Table 1 compares the features of blood glucose monitoring, and first generation CGM and FGM.

Table 1. Means for patients to monitor glucose levels at home.

\begin{tabular}{|l|l|l|l|}
\hline & Blood Glucose & RT-CGM & FGM \\
\hline Fluid tested & blood & interstitial fluid & interstitial fluid \\
\hline Frequency & $\begin{array}{l}\text { Determined by } \\
\text { user }\end{array}$ & $1-5$ minutes & $\begin{array}{l}15 \text { minutes, but } \\
\text { only revealed } \\
\text { after a swipe }\end{array}$ \\
\hline Duration & Single time point & $6-7$ days & 14 days \\
\hline $\begin{array}{l}\text { Accuracy relative to venous } \\
\text { blood levels }\end{array}$ & Best 5\% & About 10\% & About 10\% \\
\hline Calibration & Sometimes & $\begin{array}{l}\text { Yes - usually } \\
12 \text {-hourly }\end{array}$ & Factory calibrated \\
\hline Can download data for review & Yes & Yes & Yes \\
\hline $\begin{array}{l}\text { Alarms for high or low } \\
\text { glucose level }\end{array}$ & No & Yes & No \\
\hline $\begin{array}{l}\text { Can modulate insulin } \\
\text { delivery by pump }\end{array}$ & No & $\begin{array}{l}\text { Yes-some } \\
\text { models }\end{array}$ & No \\
\hline
\end{tabular}

\begin{tabular}{|l|l|l|l|}
\hline Relative Cost & + & $++*^{*}$ & $++*^{*}$ \\
\hline $\begin{array}{l}\text { Higher frequency of use } \\
\text { associated with lower } \\
\text { HbA1c levels }\end{array}$ & Yes & Yes & Yes \\
\hline $\begin{array}{l}\text { Use associated with } \\
\text { reduced hypoglycaemia }\end{array}$ & Variable & $\begin{array}{l}\text { Variable }- \text { yes if } \\
\text { linked with } \\
\text { pump with LGS } \\
\text { and PLGS }\end{array}$ & Variable \\
\hline
\end{tabular}

* less costly if subsidised, which it is in some countries for some subgroups of patient

\section{Next generation interstitial fluid glucose monitoring - implantable sensors}

The next generation of interstitial fluid glucose monitoring devices are implantable (into subcutaneous tissue), with potential to last up to $1-2$ years. The first clinically available system, whilst not yet available in Australia, is the Eversense CGM system, approved for up to 180 days use in Europe [45], and recently approved by the USA Federal Drug and Administration (FDA) for up to 90 days use [46]. The Eversense CGM system uses a small fluorescence light based sensor implanted in the upper arm under local anaesthetic via a $5-8 \mathrm{~mm}$ incision during an outpatient procedure. The user then wears a rechargeable, removable transmitter on the skin over the sensor which powers the sensor and sends a signal which converts to real-time glucose readings (every 5-minutes) on a smartphone app. The device can alert wearers to high or low glucose levels via the smartphone, and even when out of range of the smartphone by vibration of the onbody transmitter. Other devices which are fully implantable (sensor and transmitters) and contact lens type sensors are still in development.

\section{Home Monitoring Devices are Tools}

Use of any of the above glucose (or ketone) measuring devices is a tool. It is how the information they provide is used to guide drug dosing, food, physical activity and seeking medical advice that are the treatments. This requires both knowledge and its implementation. With the rapid growth in the number of people with diabetes the time available to explain new technologies to patients, to review the results and to assess their knowledge is limited. There are many issues that must be addressed during a diabetes related consultation, including control of glucose and other risk factors (such as blood pressure and lipids), lifestyle assessment and modulation, history taking, physical examinations, mental health support, ordering and interpreting test results, prescriptions and regulatory paperwork (such as for licences, insurance or travel). Any systems that can help the clinician and the person with diabetes improve their diabetes care and outcomes merit consideration.

\section{A Survey to Assess Glucose Self-Management Practice}

To assist the busy clinician to assess the glucose self-care practices of his or her adult patients with T1D we developed a paper-based survey suitable for self-administration whilst in 
the clinic waiting room. Given the frequency and risks of overnight hypoglycaemia there was an emphasis on overnight glucose control. Results have been published [47, 48]. On average the 16-question survey, available on request from the authors, took $\approx 11$ minutes to complete. The survey was conducted prior to the availability of FGM in Australia and with very few regular CGM users. We believe this is a novel tool, and may be used and adapted by other users for their practices, and to incorporate CGM and FGM use.

We demonstrated the survey feasibility and similar responses from adults with T1D in two Australian tertiary referral diabetes clinics and in a general practice (GP) clinic with a strong interest in T1D $[47,48]$. Whilst the average number of self-reported BG tests per day was above the recommended four tests per day, about one third of patients reported never testing their BG overnight, despite our clinicians usually recommending episodic overnight testing given the frequency and risks of nocturnal hypoglycaemia. In contrast $9 \%$ of patients reported testing their BG overnight every night. Such patients could benefit from CGM with alarms or use of insulin pumps with low glucose insulin suspends options. As usually recommended, to avoid nocturnal hypoglycaemia, subjects targeted higher BG levels at bedtime and overnight, but sometimes targeted levels in the teens. Targeted BG levels were similar for those reporting impaired and normal hypoglycaemia awareness. Whilst patients had adequate BG test-strips, about one third reported not having in-date ketone test-strips at home. A likely contributor may be that, in contrast to BG test-strips, ketone strips are relatively costly for the user due to lack of a subsidy by the Australian Government.

We found that suboptimal treatment of nocturnal hypoglycaemia events was common. Only $37 \%$ of the hospital clinics and $23 \%$ of the GP clinic patients reporting eating the recommended refined carbohydrate to rapidly increase BG levels followed by complex carbohydrate to prevent hypoglycaemia recurrence if the next meal was not within 20 minutes. Almost half of people reported consuming refined carbohydrate only, and often not checking BG levels, as recommended, prior to returning to sleep. This could increase nocturnal hypoglycaemia recurrence risk. The $6 \%$ of patients who reported consuming complex carbohydrate alone for hypoglycaemia may have a delayed recovery from hypoglycaemia. We speculate these behaviours may relate to a need for education, lack of nearby optimal foods, a desire to return to sleep promptly and / or to prevent posthypoglycaemia hyperglycaemia $[47,48]$.

A high percentage of survey participants reported that they would not change their overnight diabetes plan if they had extra exercise, alcohol or illness $(50 \%, 60 \%$ and $48 \%$ respectively), which may increase risk of nocturnal hypoglycaemia after exercise or alcohol, and of hyperglycaemia / ketoacidosis during illness. Many reported a lack of confidence in adjusting their diabetes care plans.

Patients were asked how they would treat pre-bed BG levels of $4,8,11,15 \mathrm{mmol}, 18 \mathrm{mmol}$ with moderate ketones and $20 \mathrm{mmol} / \mathrm{l}$. Less than one in four patients always suggested safe responses to these hypothetical bed-time BG levels. Mid-range levels, where no action was usually needed, had the highest rates of safe responses $[47,48]$. There is substantial evidence that education is key in improving diabetes management, especially in reducing hypoglycaemia [49-52].

Many patients (33\%) desired further diabetes education, which was provided in this study, but there were few demographic factors to predict who might require or want education. The survey we developed and utilised, which could be incorporated into an annual review, perhaps associated with risk factor and complication screening, may assist. With appropriate modifications the survey could be administered electronically, in other languages, and to paediatric / adolescent patients and their carers.

There are well recognised limitations of self-reporting in surveys. Whilst participants may report what they perceive to be desired actions, rather than actual behaviour, nevertheless, there are still high rates of suboptimal answers in our surveys. There may also be recall bias. Selection bias risk was small due to very high rates of patient participation. We recognise that the suboptimal answers may reflect real knowledge gaps or may reflect lack of implementation of knowledge.

\section{Diabetes Knowledge and its Implementation}

There is much to learn about living with diabetes, and education should be regarded as an ongoing process and a shared responsibility of the clinician and the patient. Time and tools to assess knowledge are important, and the availability of new home-monitoring systems, as described above, increases the time and knowledge base required, for both clinicians and users.

There are many sources of diabetes education and they may sometimes provide contrasting information. In addition to the various clinicians in the diabetes care team, information may be obtained from family, friends, other people with diabetes, support groups, industry, literature and the internet. There is the potential for both valuable and misleading information. Clinicians should be aware of suitable information sources and websites to recommend, and be well-prepared to discuss divergent information. Websites by national diabetes organisations and the Juvenile Diabetes Research Foundation are usually reputable, evidence-based and up-to-date.

Although the care of and outcomes for patients with T1D has improved immeasurably in recent times, many challenges remain. Barriers to optimal management of diabetes may be broadly thought of as patient related, clinician related and system related. Managing and living with T1D is hard work, as summarised wryly in a type 1 meme: "Type 1 diabetes is a fulltime job that we didn't apply for, don't want and can't resign from, and there's no vacation or pay" [53]. The relentless nature of T1D can lead to diabetes distress, depression and burn-out, which can lead to a relegation of diabetes management in the 
hierarchy of priorities for the person with diabetes [10, 54-56]. As a result, we often witness both the expected mechanics and consequences of poorer self-management.Commonmanifestations include infrequent glucose or ketone monitoring ("I don't want to know"), and sub-optimal treatment ("I don't want to do") including 'guesstimated' or omitted doses of insulin. This can result in poorer glycaemic control, guilt, avoidance and depression. Thus, the struggling individual battling for control over their T1D can enter a negative spiral.

T1D imposes significant financial costs on the person with diabetes and their families [57], particularly if care is not subsidised by the healthcare system. In addition to the cost of medications there are the costs associated with the various paraphernalia used to treat and monitor their diabetes such as syringes, pumps, CGM, FGM, BG meters, glucose and ketone test-strips. Less visible is the cost in time, effort and avoidance of otherwise pleasurable activities and / or healthy activities, such as exercise, due to fear of hypoglycaemia, hyperglycaemia or embarrassment [58].

Poor health literacy has been identified as a significant predictor of poorer clinical outcomes in many conditions, including diabetes [59-62]. People with diabetes may not always fully appreciate the gravity of their situation or they may struggle with the complexity of the pathophysiology of their disease and its management. This may change throughout their life course with T1D, such as related to their age and cognitive ability, development of diabetes complications, or the use of modern diabetes technologies, such as insulin pumps and insulin bolus calculators. Numeracy and literacy may be inadequate for the task of carbohydrate counting and insulin dosage calculations. Patients may experience loss of control, feelings of inadequacy and a sense of failure, adding to the challenge and burden of self-management.

Clinician barriers, particularly in the primary care sector, may relate to lack of knowledge, time, experience and confidence dealing with T1D. Health care practitioners may misinterpret a patient's poor outcomes as a lack of interest, laziness or obstinacy, rather than picking up on their patient's distress and supporting them appropriately. There are also clinician and healthcare system barriers to acquiring and implementing relevant T1D related knowledge, including that related to home glucose and ketone monitoring. Clinicians may themselves be stressed, time-poor and burnt out. The resulting maladaptation is to accept the status quo, resulting in therapeutic inertia, allowing a toxic situation, such as high $\mathrm{HbA} 1 \mathrm{c}$ levels, frequent hypoglycaemia events and high GV to persist. System related barriers may relate to workforce shortages, proximity to care and hours that the T1D service may be offered. When demands exceed resources, distress and maladaptive behaviour can ensue in both patients and their clinicians.

\section{Conclusions}

The importance of good glucose control in people with T1D is well-recognised. There is an increasing array of tools available to assess glucose and ketones at home, which have proven benefit in clinical trials. Cost is often a barrier to their regular use. Patients and their clinicians must also have adequate knowledge, time to acquire it, and most importantly the ability, finances and desire to implement it appropriately in order to maximise the clinical benefits.

\section{Conflict of Interest}

AJJ and DNO have received peer reviewed research grants from Medtronic and are on advisory boards for Medtronic (Australia) and Abbott (Diabetes). GK, ES, GRF and ASJ have no conflict of interest.

None of the authors have stocks or shares of any companies products which are cited in this publication.

\section{References}

1. Cho NH, Shaw JE, Karuranga S, et al. IDF Diabetes Atlas: Global estimates of diabetes prevalence for 2017 and projections for 2045. Diabetes Res Clin Pract. 2018; 138: 271-281. doi: 10.1016/j.diabres.2018.02.023

2. Weng J, Zhou Z, Guo L, et al. Incidence of type 1 diabetes in China, 201013: population based study. BMJ. 2018; 360: 5295. doi: 10.1136/bmj.j5295

3. Gubitosi-Klug RA, Group DER. The diabetes control and complications trial/epidemiology of diabetes interventions and complications study at 30 years: summary and future directions. Diabetes Care. 2014; 37(1): 44 49. doi: $10.2337 / \mathrm{dc} 13-2148$

4. Diabetes $C$, Complications Trial/Epidemiology of Diabetes I, Complications Research G, Lachin JM, White NH, Hainsworth DP, et al. Effect of intensive diabetes therapy on the progression of diabetic retinopathy in patients with type 1 diabetes: 18 years of follow-up in the DCCT/EDIC. Diabetes. 2015; 64(2): 631-642. doi: 10.2337/db14-0930

5. White NH, Sun W, Cleary PA, et al. Prolonged effect of intensive therapy on the risk of retinopathy complications in patients with type 1 diabetes mellitus: 10 years after the Diabetes Control and Complications Trial. Arch Ophthalmol. 2008; 126(12): 1707-1715. doi: 10.1001/archopht.126.12.1707

6. American Diabetes Association. 6. Glycemic Targets: Standards of Medical Care in Diabetes-2018. Diabetes Care. 2018; 41(1): 55-64. doi: 10.2337/dc18-S006

7. Cheung NW, Conn JJ, d'Emden MC, et al. Position statement of the Australian Diabetes Society: individualisation of glycated haemoglobin targets for adults with diabetes mellitus. Med J Aust. 2009; 191(6): 339344.

8. National Association of Diabetes Centers. Final Report ANDIAB 2011. [Available from: https://www.adea.com.au/wp-content/uploads/2013/08/ ANDIAB-Final-Report-2011.pdf. Accessed: 28 May 2018.

9. Murata T, Tsuzaki K, Yoshioka F, et al. The relationship between the frequency of self-monitoring of blood glucose and glycemic control in patients with type 1 diabetes mellitus on continuous subcutaneous insulin infusion or on multiple daily injections. J Diabetes Investig. 2015; 6(6): 687691. doi: $10.1111 /$ jdi.12362

10. Hendrieckx C, Halliday JA, Bowden JP, et al. Severe hypoglycaemia and its association with psychological well-being in Australian adults with type 1 diabetes attending specialist tertiary clinics. Diabetes Res Clin Pract. 2014; 103(3): 430-436. doi: 10.1016/j.diabres.2013.12.005

11. Ly TT, Gallego PH, Davis EA, Jones TW. Impaired awareness of hypoglycemia in a population-based sample of children and adolescents with type 1 diabetes. Diabetes Care. 2009; 32(10): 1802-1806. doi: 10.2337/dc09-0541

12. Jones TW, Porter $P$, Sherwin RS, et al. Decreased epinephrine responses to hypoglycemia during sleep. N Engl J Med. 1998; 338(23): 1657-1662. doi: 10.1056/NEJM199806043382303

13. Hsieh A, Twigg SM. The enigma of the dead-in-bed syndrome: challenges in predicting and preventing this devastating complication of type 1 diabetes. J Diabetes Complications. 2014; 28(5): 585-587. doi: 10.1016/j. jdiacomp.2014.04.005

14. Tu E, Twigg SM, Semsarian C. Sudden death in type 1 diabetes: the mystery of the 'dead in bed' syndrome. Int J Cardiol. 2010; 138(1): 91-93. doi: 10.1016/j.ijcard.2008.06.021 
15. Novodvorsky P, Bernjak A, Chow E, et al. Diurnal Differences in Risk of Cardiac Arrhythmias During Spontaneous Hypoglycemia in Young People With Type 1 Diabetes. Diabetes Care. 2017; 40(5): 655-662. doi: 10.2337/ dc16-2177

16. Ayano-Takahara S, Ikeda K, Fujimoto $S$, et al. Glycemic variability is associated with quality of life and treatment satisfaction in patients with type 1 diabetes. Diabetes Care. 2015; 38(1): 1-2. doi: 10.2337/dc14-1801

17. Kilpatrick ES, Rigby AS, Goode K, Atkin SL. Relating mean blood glucose and glucose variability to the risk of multiple episodes of hypoglycaemia in type 1 diabetes. Diabetologia. 2007; 50(12): 2553-2561. doi: 10.1007/ s00125-007-0820-z

18. Kovatchev BP. Metrics for glycaemic control - from $\mathrm{HbA} 1 \mathrm{c}$ to continuous glucose monitoring. Nat Rev Endocrinol. 2017; 13(7): 425-436. doi: 10.1038/nrendo.2017.3

19. Monnier L, Colette C, Wojtusciszyn A, et al. Toward Defining the Threshold Between Low and High Glucose Variability in Diabetes. Diabetes Care. 2017; 40(7): 832-838. doi: 10.2337/dc16-1769

20. National Glycohemoglobin Standardization Program. Factors that interfere with HbA1c test results. 2018 [Available from: http://www.ngsp. org/factors.asp]. Accessed: 28 May 2018.

21. Telo GH, de Souza MS, Andrade TS, Schaan BD. Comparison between adherence assessments and blood glucose monitoring measures to predict glycemic control in adults with type 1 diabetes: a cross-sectional study. Diabetol Metab Syndr. 2016; 8: 54. doi: 10.1186/s13098-016-0162-4

22. Australian Diabetes Educators Association. Clinical Guiding Principles for Sick Day Management of Adults with Type 1 and Type 2 Diabetes. Technical document. Canberra: Australian Diabetes Educators Association; 2016 [Available from: https://www.adea.com.au/wp-content/uploads/2009/10/ Clinical-Guiding-Principles-for-Sick-Day-Management-of-Adults-with-type-1and-type-2-diabetes-Review-2016.docx.pdf ]. Accessed: 28 May 2018.

23. Johansen K, Svendsen PA, Lorup B. Variations in renal threshold for glucose in Type 1 (insulin-dependent) diabetes mellitus. Diabetologia. 1984; 26(3): 180-182.

24. Danne T, Nimri R, Battelino $T$, et al. International Consensus on Use of Continuous Glucose Monitoring. Diabetes Care. 2017; 40(12): 1631-1640. doi: $10.2337 / \mathrm{dc} 17-1600$

25. Klonoff DC, Ahn D, Drincic A. Continuous glucose monitoring: A review of the technology and clinical use. Diabetes Res Clin Pract. 2017; 133: 178192. doi: 10.1016/j.diabres.2017.08.005

26. Petrie JR, Peters AL, Bergenstal RM, Holl RW, Fleming GA, Heinemann L. Improving the Clinical Value and Utility of CGM Systems: Issues and Recommendations: A Joint Statement of the European Association for the Study of Diabetes and the American Diabetes Association Diabetes Technology Working Group. Diabetes Care. 2017; 40(12): 1614-1621. doi: 10.2337/dci17-0043

27. Slattery D, Choudhary P. Clinical Use of Continuous Glucose Monitoring in Adults with Type 1 Diabetes. Diabetes Technol Ther. 2017; 19(2): 55-61. doi: 10.1089/dia.2017.0051

28. Wood A, O'Neal D, Furler J, Ekinci El. Continuous glucose monitoring: a review of the evidence, opportunities for future use and ongoing challenges. Intern Med J. 2018; 48(5): 499-508. doi: 10.1111/imj.13770

29. Szypowska A, Ramotowska A, Dzygalo K, Golicki D. Beneficial effect of realtime continuous glucose monitoring system on glycemic control in type 1 diabetic patients: systematic review and meta-analysis of randomized trials. Eur J Endocrinol. 2012; 166(4): 567-574. doi: 10.1530/EJE-11-0642

30. Vigersky R, Shrivastav M. Role of continuous glucose monitoring for type 2 in diabetes management and research. J Diabetes Complications. 2017; 31(1): 280-287. doi: 10.1016/j.jdiacomp.2016.10.007

31. McLachlan $\mathrm{K}$, Jenkins $A, O^{\prime} N$ eal $D$. The role of continuous glucose monitoring in clinical decision-making in diabetes in pregnancy. Aust $N \mathrm{Z} J$ Obstet Gynaecol. 2007; 47(3): 186-190. doi: 10.1111/j.1479-828X.2007.00716.x

32. Abraham MB, Nicholas JA, Smith GJ, et al. Reduction in Hypoglycemia with the Predictive Low-Glucose Management System: A Long-term Randomized Controlled Trial in Adolescents with Type 1 Diabetes. Diabetes Care. 2018; 41(2): 303-310. doi: 10.2337/dc17-1604
33. Bergenstal RM, Klonoff DC, Garg SK, et al. Threshold- based insulin-pump interruption for reduction of hypoglycemia. N Engl J Med. 2013; 369(3): 224-232. doi: 10.1056/NEJMoa1303576

34. Ly TT, Nicholas JA, Retterath A, Lim EM, Davis EA, Jones TW. Effect of sensor- augmented insulin pump therapy and automated insulin suspension vs standard insulin pump therapy on hypoglycemia in patients with type 1 diabetes: a randomized clinical trial. JAMA. 2013; 310(12): 1240-1247. doi: 10.1001/jama.2013.277818

35. Feig DS, Donovan LE, Corcoy $\mathrm{R}$, et al. Continuous glucose monitoring in pregnant women with type 1 diabetes (CONCEPTT): a multicentre international randomised controlled trial. Lancet. 2017; 390(10110): 23472359. doi: 10.1016/S0140-6736(17)32400-5

36. Garg SK, Weinzimer SA, Tamborlane WV, Buckingham BA, Bode BW, Bailey TS, et al. Glucose Outcomes with the In-Home Use of a Hybrid Closed-Loop Insulin Delivery System in Adolescents and Adults with Type 1 Diabetes. Diabetes Technol Ther. 2017; 19(3): 155-163. doi: 10.1089/ dia.2016.0421

37. Weisman A, Bai JW, Cardinez M, Kramer CK, Perkins BA. Effect of artificial pancreas systems on glycaemic control in patients with type 1 diabetes: a systematic review and meta- analysis of outpatient randomised controlled trials. Lancet Diabetes Endocrinol. 2017; 5(7): 501-512. doi: 10.1016/S22138587(17)30167-5

38. Al Hayek AA, Robert AA, Al Dawish MA. Evaluation of FreeStyle Libre Flash Glucose Monitoring System on Glycemic Control, Health-Related Quality of Life, and Fear of Hypoglycemia in Patients with Type 1 Diabetes. Clin Med Insights Endocrinol Diabetes. 2017; 10: 1179551417746957. doi: 10.1177/1179551417746957

39. Leelarathna L, Wilmot EG. Flash forward: a review of flash glucose monitoring. Diabet Med. 2018; 35(4): 472-482. doi: 10.1111/dme.13584

40. Oskarsson P, Antuna R, Geelhoed-Duijvestijn P, Krger J, Weitgasser R, Bolinder J. Impact of flash glucose monitoring on hypoglycaemia in adults with type 1 diabetes managed with multiple daily injection therapy: a prespecified subgroup analysis of the IMPACT randomised controlled trial. Diabetologia. 2018; 61(3): 539-550. doi: 10.1007/s00125-017-4527-5

41. Fokkert MJ, van Dijk PR, Edens MA, et al. Performance of the FreeStyle Libre Flash glucose monitoring system in patients with type 1 and 2 diabetes mellitus. BMJ Open Diabetes Res Care. 2017; 5(1): 000320. doi: 10.1136/bmjdrc-2016-000320

42. Dunn TC, Xu Y, Hayter G, Ajjan RA. Real-world flash glucose monitoring patterns and associations between self-monitoring frequency and glycaemic measures: A European analysis of over 60 million glucose tests. Diabetes Res Clin Pract. 2018; 137:37-46. doi: 10.1016/j.diabres.2017.12.015

43. Maahs DM, DeSalvo $D$, Pyle $L$, et al. Effect of acetaminophen on CGM glucose in an outpatient setting. Diabetes Care. 2015; 38(10): 158-159. doi: $10.2337 /$ dc15-1096

44. FreeStyle Libre. Frequently asked questions about the FreeStyle Libre 14 day System. 2018.

45. BusinessWire. Senseonics Announces CE Mark Approval for Eversense ${ }^{\circledR}$ XL CGM System. 2017.

46. FDA. FDA approves first continuous glucose monitoring system with a fully implantable glucose sensor and compatible mobile app for adults with diabetes. US Food and Drug Administration. 2018.

47. Kilov G, Januszewski AS, McGrath RT, et al. Comparable glucose related self-care by adults with Type 1 diabetes in primary-care and specialist diabetes clinics and need for patient education. Diabetes \& Primary Care Aust. 2018.

48. Larsson CR, Januszewski AS, McGrath RT, et al. Suboptimal behaviour and knowledge regarding overnight glycaemia in adults with Type 1 diabetes is common. Intern Med J. 2018; doi: 10.1111/imj.13798

49. Elliott J, Jacques RM, Kruger J, et al. Substantial reductions in the number of diabetic ketoacidosis and severe hypoglycaemia episodes requiring emergency treatment lead to reduced costs after structured education in adults with Type 1 diabetes. Diabet Med. 2014; 31(7): 847-853. doi: 10.1111/dme. 12441 
50. Hopkins $D$, Lawrence I, Mansell $P$, et al. Improved biomedical and psychological outcomes 1 year after structured education in flexible insulin therapy for people with type 1 diabetes: the U.K. DAFNE experience. Diabetes Care. 2012; 35(8): 1638-1642. doi: 10.2337/dc11-1579

51. Muhlhauser $\mathrm{I}$, Jorgens $\mathrm{V}$, Berger $\mathrm{M}$, et al. Bicentric evaluation of a teaching and treatment programme for type 1 (insulin-dependent) diabetic patients: improvement of metabolic control and other measures of diabetes care for up to 22 months. Diabetologia. 1983; 25(6): 470-476.

52. Plank J, Kohler G, Rakovac I, et al. Long-term evaluation of a structured outpatient education programme for intensified insulin therapy in patients with Type 1 diabetes: a 12-year follow-up. Diabetologia. 2004; 47(8): 13701375. doi: 10.1007/s00125-004-1456-x

53. Unknown Author. Depression and its relationship to Type 1 diabetes [Available from: https://beyondtype1.org/depression-and-its-relationship-totype-1/ ]. Accessed: 28 May 2018.

54. Dennick K, Sturt J, Speight J. What is diabetes distress and how can we measure it? Anarrative review and conceptual model. I Diabetes Complications. 2017; 31(5): 898-911. doi: 10.1016/j.jdiacomp.2016.12.018

55. Fenwick EK, Rees G, Holmes-Truscott E, Browne JL, Pouwer F, Speight J. What is the best measure for assessing diabetes distress? A comparison of the Problem Areas in Diabetes and Diabetes Distress Scale: results from Diabetes MILES-Australia. J Health Psychol. 2018; 23(5): 667-680. doi: $10.1177 / 1359105316642006$
56. Speight J, Holmes-Truscott E, Harvey DM, et al. Structured type 1 diabetes education delivered in routine care in Australia reduces diabetes-related emergencies and severe diabetes-related distress: The OzDAFNE program. Diabetes Res Clin Pract. 2016; 112: 65-72. doi: 10.1016/j. diabres.2015.11.002

57. Ogle GD, Kim H, Middlehurst AC, Silink $M$, Jenkins AJ. Financial costs for families of children with Type 1 diabetes in lower-income countries. Diabet Med. 2016; 33(6): 820-826. doi: 10.1111/dme.12997

58. Martyn-Nemeth P, Quinn L, Penckofer S, Park C, Hofer V, Burke L. Fear of hypoglycemia: Influence on glycemic variability and self-management behavior in young adults with type 1 diabetes. J Diabetes Complications. 2017; 31(4): 735-741. doi: 10.1016/j.jdiacomp.2016.12.015

59. Bailey SC, Brega AG, Crutchfield TM, et al. Update on health literacy and diabetes. Diabetes Educ. 2014; 40(5): 581-604. doi: 10.1177/0145721714540220

60. Boren SA. A review of health literacy and diabetes: opportunities for technology. J Diabetes Sci Technol. 2009; 3(1): 202-209. doi: 10.1177/193229680900300124

61. Kim SH, Lee A. Health-Literacy-Sensitive Diabetes Self-Management Interventions: A Systematic Review and Meta-Analysis. Worldviews Evid Based Nurs. 2016; 13(4): 324-333. doi: 10.1111/wvn.12157

62. Yee LM, Niznik CM, Simon MA. Examining the Role of Health Literacy in Optimizing the Care of Pregnant Women with Diabetes. Am J Perinatol. 2016; 33(13): 1242-1249. doi: 10.1055/s-0036-1584540 